\title{
Towards Increased Concentration Sensitivity for Continuous Wave EPR Investigations of Spin-Labeled Biological Macromolecules at High Fields
}

Likai Song ${ }^{\mathrm{a}, \mathrm{e}}$, Zhanglong Liu $^{\mathrm{c}, \mathrm{e}}$, Pavanjeet Kaur ${ }^{\mathrm{a}, \mathrm{b}, \mathrm{e}}$, Jackie M. Esquiaqui ${ }^{\mathrm{c}}$, Robert I. Hunter ${ }^{\mathrm{d}}$, Stephen Hill ${ }^{\mathrm{a}, \mathrm{b}}$, Graham M. Smith ${ }^{\mathrm{d}}$ and Gail E. Fanucci ${ }^{\mathrm{c}, *}$

${ }^{a}$ National High Magnetic Field Laboratory and ${ }^{b}$ Department of Physics, Florida State University, Tallahassee, FL 32310, USA

${ }^{c}$ Department of Chemistry, University of Florida, PO Box 117200, Gainesville, FL 32611, USA

${ }^{\mathrm{d}}$ School of Physics and Astronomy, University of St. Andrews, North Haugh, St. Andrews KY16 9SS, United Kingdom

${ }^{\mathrm{e}}$ These authors contributed equally to this work.

*To whom correspondence may be addressed. E-mail: fanucci@chem.ufl.edu

\begin{abstract}
High-field, high-frequency electron paramagnetic resonance (EPR) spectroscopy at W$(\sim 95 \mathrm{GHz})$ and D-band $(\sim 140 \mathrm{GHz})$ is important for investigating the conformational dynamics of flexible biological macromolecules because this frequency range has increased spectral sensitivity to nitroxide motion over the 100 ps to 2 ns regime. However, low concentration sensitivity remains a roadblock for studying aqueous samples at high magnetic fields. Here, we examine the sensitivity of a non-resonant thin-layer cylindrical sample holder, coupled to a quasi-optical induction-mode W-band EPR spectrometer (HiPER), for continuous wave (CW) EPR analyses of: (i) the aqueous nitroxide standard, TEMPO; (ii) the unstructured to $\alpha$-helical transition of a model IDP protein; and (iii) the base-stacking transition in a kink-turn motif of a large $232 \mathrm{nt}$ RNA. For sample volumes of $\sim 50 \mu \mathrm{L}$, concentration sensitivities of $2-20 \mu \mathrm{M}$ were achieved, representing a $\sim 10$-fold enhancement compared to a cylindrical $\mathrm{TE}_{011}$ resonator on a commercial Bruker W-band spectrometer. These results therefore highlight the sensitivity of the thin-layer sample holders employed in HiPER for spin-labeling studies of biological macromolecules at high fields, where applications can extend to other systems that are facilitated by the modest sample volumes and ease of sample loading and geometry.
\end{abstract}




\section{Introduction}

Structure and structure-function relationships of macromolecules are areas of intense EPR effort [1-16]. Coupled with site-directed spin-labeling (SDSL), EPR is oftentimes used to characterize protein and nucleic acid structures and dynamics, conformational changes, molecule folding, macromolecule complexes, and oligomeric structures [1-15, 17]. The majority of biomolecules do not contain unpaired electrons from which one can obtain an EPR signal; therefore, spin-labeling approaches have been developed [15, 18-25] where site-specific persistent radicals or paramagnetic metal-probes are incorporated at specific locations within a biomolecule. Properties of the EPR spectra that originate from these probes, positioned at welldefined vantage points, provide structural and dynamic constraints. High-field EPR approaches offer the possibility of increased signal sensitivity and information content, thus potentially extending the scope of research aimed at biological macromolecules. High fields increase EPR sensitivity and broaden its practice through improved g-factor resolution for extracting biophysical constraints, which include topics such as dynamics of molecular motion, local polarity and hydrogen bonding, relative orientation information, and membrane ordering [26-33]. Moreover, multi-frequency EPR is especially powerful for describing complex local biophysical dynamics [34-36].

With regards to applications in studying macromolecular conformational dynamics and flexibility, the increased Larmor frequency of high-field EPR allows for a "shift" in the motional averaging timescale towards fast motion. Traditionally, X-band $(9.5 \mathrm{GHz})$ EPR spectroscopy has been utilized for SDSL investigations of biological macromolecules. At W-band ( $94 \mathrm{GHz})$, the sensitivity to intermediate motion shifts to a time regime of faster motion with smaller correlation times, which affords increased spectral sensitivity to motions that appear in the "fast-motion" limit at X-band. Examples of nitroxide spin-labeled molecules falling into a fast motion regime (i.e. correlation times $<2 \mathrm{~ns}$ ) include small peptides/proteins, small nucleic acids, unstructured proteins/protein segments, and backbone/nucleobase labeling of RNAs [37-42]. For these biological systems, it is oftentimes challenging to extract dynamic parameters from the subtle differences of the narrow line shapes of CW EPR spectra at X-band. Consequently, in this time regime, molecular motion nearly completely averages the g-tensor and hyperfine-tensor anisotropy. The resulting narrow line shapes show only slight changes in 
empirical mobility parameters, such as the central line width $\left(\Delta H_{\mathrm{o}}\right)$ and the inverse of the second moment $\left(\left\langle H^{2}\right\rangle^{-1}\right)$. Alternatively, high-field EPR spectra obtained at W-band provide "sweetspots" with enhanced line shape sensitivity for motions with correlation times ranging over 0.1 $2 \mathrm{~ns}$ [43].

A prime example of X-band EPR studies that fall within this "fast-motion" regime is the characterization of dynamics of intrinsically disordered proteins (IDPs) [37]. IDPs are described as proteins or protein segments that lack a well-defined secondary or tertiary structure [44-48]. IDPs often exist in different structural states in vivo, from disordered to fully structured; and carry out their function either through a structural transition between different states or through their disordered flexible structure. IDPs oftentimes play key roles in cellular activity, such as transcription and translation regulation, signal transduction, post-translation modification, cellular transport, and protein assembly [45, 49]. Structural and dynamic investigation of IDPs is of particular interest to elucidate their structure-function relationships.

Concentration sensitivity is a challenge for high-field EPR studies of biologically relevant samples in non-frozen aqueous solutions. This stems primarily from the shorter wavelengths associated with the higher microwave frequencies. First and foremost, if using a resonator, both the dimensions of the resonant structure and the corresponding active volume associated with the microwave $B_{1}$-field maximum ( $E$-field minimum) shrink appreciably at high fields. This is particularly important for studies of aqueous samples because of the need to minimize dielectric losses in the solution due to interaction with the electric ( $E$-field) component of the microwaves. For a typical enclosed cavity, this usually requires shrinking the sample holder in at least two dimensions. At X-band (wavelength $=3.3 \mathrm{~cm}$ ), capillaries with $\sim 1 \mathrm{~mm}$ inner diameter (I.D.) have an active volume in the 3-10 $\mu \mathrm{L}$ range, whereas capillaries of 0.1-0.2 mm I.D. present a volume of only $\sim 0.1 \mu \mathrm{L}$ in a typical Bruker W-band (wavelength $=3.3 \mathrm{~mm}$ ) cylindrical cavity. This reduction in sample volume oftentimes results in decreased concentration sensitivity at $\mathrm{W}$ band, relative to $\mathrm{X}$-band, even though the absolute spin sensitivity is enhanced due to increased magnetization at high field. Even when using a quasi-optical approach with no resonator, as in the present study, or even with a Fabry-Pérot resonator [50], the reduction in penetration depth with increasing frequency also results in an unavoidable decrease in active sample volume. Moreover, broader spectral lines typically observed for nitroxides at high fields lead to further 
reductions in overall sensitivity. Therefore, concentration sensitivity remains a major problem for CW EPR studies at high fields, with technological challenges limiting widespread applications.

A recently developed quasi-optical $94 \mathrm{GHz}$ EPR spectrometer (HiPER) provides exceptional sensitivity and time resolution $[51,52]$. For instance, it has demonstrated $\sim 1 \mu \mathrm{M}$ concentration sensitivity for pulsed dipolar EPR, or DEER (Double Electron-Electron Resonance), owing to its unique quasi-optical inductive-mode design that gives exceptional cross-polar isolation, its high power (kilowatt), high instantaneous bandwidth pulses, and the possibility to use non-resonant sample holders of large volume [52].

The present study examines the concentration sensitivity of several newly developed nonresonant thin-layer cylindrical sample holders, designed specifically for low-power CW EPR studies of aqueous samples using HiPER. We first assess the sensitivities of these holders using the radical TEMPO as a standard. We then evaluate the performance of an optimized sample holder, with a $\sim 50 \mu \mathrm{L}$ sample volume, by collecting W-band EPR spectra reflective of the unstructured to helical transition of an intrinsically disordered protein $\mathrm{IA}_{3}$. We also demonstrate a second application involving characterization of the base dynamics in large RNAs. A 10-fold enhancement in concentration sensitivity is achieved using these thin-layer sample holders with HiPER compared to a $\mathrm{TE}_{011}$ cylindrical cavity on a commercial Bruker W-band spectrometer. This study therefore highlights the advantages of using non-resonant induction mode sample holders for EPR studies of low concentration aqueous solutions at high magnetic fields, and their application to fast-motion biological samples.

\section{Materials and Methods}

\section{1. Materials}

3-(2-iodoacetamido)-PROXYL (IAP) spin labels and 2,2,6,6-Tetramethyl-1-piperidinyloxy (TEMPO) were purchased from Sigma Aldrich (St. Louis, MO). TEMPO solutions for EPR measurements were prepared in autoclaved deionized water (Barnstead NANOpure, CA). The primers for site-directed mutagenesis were purchased from Integrated DNA Technologies (Coralville, IA). E. coli codon-optimized DNA fragment for encoding the inhibitor $\left(\mathrm{IA}_{3}\right)$ of yeast 
proteinase A (YprA) was purchased from DNA2.0 (Menlo Park, CA) and the corresponding protein sequence is shown in supplementary materials. Cysteine was introduced to Glu-10 via site-directed mutagenesis by using polymerase chain reaction (PCR) with the assistance of the custom designed primers. The $\mathrm{IA}_{3}$ gene contained 8 additional codons which could be translated into two linking amino acids leucine and glutamic acid (LE) and a 6xHis-tag. The gene was then cloned into the pET-22b vector by two restriction enzyme digestion sites. The DNA sequence of $\mathrm{IA}_{3}$ in this article was confirmed with Sanger sequencing in the Interdisciplinary Center for Biotechnology Research in the University of Florida. BL21 (DE3) PlysS cells were purchased from Invitrogen (Carlsbad, CA). Synthetic RNA fragments containing 4-thiouridine modified nucleobases were purchased from Dharmacon (Pittsburgh, PA). $0.15 \mathrm{~mm}$ inner diameter quartz capillary tubes for W-band Bruker EPR measurements were purchased from VitroCom (Mountain Lakes, NJ). All other reagents were purchased from Fisher Scientific (Pittsburgh, PA) unless otherwise indicated.

\section{2. $\mathrm{IA}_{3}$ Protein Expression and Purification}

Protein expression, purification and spin-labeling of the E10C $\mathrm{IA}_{3}$ construct proceeded as described previously [37, 43]. Briefly, protein expression was induced by Isopropyl- $\beta$-Dthiogalactopyranoside (IPTG); which was optimized by pilot expression and monitoring the intensity of the target protein band intensity in sodium dodecyl sulfate (SDS) polyacrylamide gel electrophoresis (PAGE). Afterwards, the cell lysate was boiled in water and centrifuged to remove precipitants. The soluble supernatant was loaded into the nickel affinity column (GE Healthcare Life Sciences, Pittsburgh, PA) for further purifications. The residual nickel ion from the column was removed by buffer exchange with the addition of EDTA (1 mM final concentration). Finally, cysteine reduction was ensured by addition of dithiothreitol (DTT, 10 $\mathrm{mM}$ final concentration) for overnight reaction at $4{ }^{\circ} \mathrm{C}$.

\subsection{Spin-Labeled $\mathrm{IA}_{3}$ Sample Preparation}

DTT was removed from the E10C IA 3 sample by buffer exchange into phosphate buffer $(50 \mathrm{~m} M$ sodium phosphate, $300 \mathrm{~m} M$ sodium chloride and $\mathrm{pH}$ 7.4) by using a HiPrep 26/10 desalting column (GE Healthcare Life Sciences, Pittsburgh, PA). Firstly, IAP spin label was dissolved in ethanol, then was added to the protein solution with 10:1 molar ratio and allowed to react 
overnight. Excess spin label was removed by buffer exchange with the same desalting column and phosphate buffer as described above. If necessary, spin-labeled protein samples were concentrated to a final concentration by using a Millipore 5,000 MWCO filter operated under gas pressure. Each individual sample contained a final volume of $1000 \mu L$, which included $600 \mu L$ protein sample in phosphate buffer $(50 \mathrm{~m} M$ sodium phosphate, $300 \mathrm{~m} M$ sodium chloride and $\mathrm{pH}$ 7.4) and corresponding volume of TFE and de-ionized water to keep all samples at equal ionic strength and $\mathrm{pH}$.

\subsection{RNA Sample Preparation}

The 232 nt spin labeled Vibrio cholerae (VC) glycine riboswitch RNA was produced utilizing our previously published protocol that employs optimized splinted ligation with T4 DNA ligase[53, 54]. Briefly described here, a short (20 nt) synthetic RNA fragment with a site specific 4-thiouridine modified nucleobase was purchased and deprotected according to the vendor's instructions. Spin labeling of the synthetic RNA fragment with IAP was performed in accordance with a previously published protocol [55]. IAP was dissolved in 83\% (100 mM) sodium phosphate buffer ( $\mathrm{pH}$ 8), 7\% ethanol, 10\% dimethylformamide and then added in excess to the deprotected synthetic RNA fragment in a 1:200 mole ratio and incubated in the dark, at room temperature, for 24 hours, and with constant rotation. After phenol/choroform/isoamyl alcohol (PCA) (25:24:1 mixture, $\mathrm{pH}$ 6.7/8.0) extraction and ethanol precipitation, the spin labeled RNA fragment was ligated to the remaining $212 \mathrm{nt}$ riboswitch RNA sequence, which was generated via in vitro transcription using T7 RNA polymerase. All details regarding transcription and ligation procedures were followed as described previously [53, 54]. The full length 232 nt, ligated, spin labeled, RNA was purified from unligated product via $6 \%$ denaturing polyacrylamide gel electrophoresis and was electroeluted overnight in Tris-EDTA (TE) buffer at $4^{\circ} \mathrm{C}$, using $150 \mathrm{~V}$, for 12-16 hours. After PCA extraction and ethanol precipitation, the final purified RNA was dissolved in water. Two $60 \mu \mathrm{M}$ RNA samples were prepared and loaded into sample holder H2 (50 $\mu \mathrm{L}$ holder): RNA in water alone, and RNA with $100 \mathrm{mM} \mathrm{KCl,} 5 \mathrm{mM}$ $\mathrm{MgCl}_{2}$, and $5 \mathrm{mM}$ glycine. 


\subsection{EPR Measurements}

High-field EPR experiments were carried out on HiPER [52] and a Bruker E680 W-band spectrometer (Billerica, MA) at the National High Magnetic Field Laboratory (NHMFL) in

Tallahassee, Florida. The architecture of the HiPER spectrometer is already published and partly described in the supplementary materials in Figure S1. Quasi-optical CW measurements were performed on HiPER [51, 52] using non-resonant induction-mode thin-layer sample holders (described in the following sections) in which the EPR signal is detected in the polarization orthogonal to the incident microwaves [the incoming linearly polarized state may be thought of as a superposition of counter-rotating circularly polarized signals, only one of which interacts resonantly with the sample, thereby generating an elliptical state with an orthogonal linear component]. The sample temperature was regulated using a CF1200 helium flow cryostat (Oxford Instruments, UK). For all HiPER experiments, the variable attenuator was set to $0 \mathrm{~dB}$, giving a maximum source power of $\sim 250 \mathrm{~mW}$ at $94 \mathrm{GHz}$. We estimate about $3 \mathrm{~dB}$ of loss from waveguide components and a further $3 \mathrm{~dB}$ of loss from the optics, giving an estimated power of $\sim 50-80 \mathrm{~mW}$ at the sample. No spectral saturation was apparent with this set up. Spectra were collected with $800 \mathrm{~Hz}$ modulation frequency, 1-4 Gauss (G) modulation amplitude, and 150$200 \mathrm{G}$ scan range, with other parameters that were optimized for each sample.

CW EPR measurements on the Bruker E680 spectrometer were conducted with a Bruker cylindrical $\mathrm{TE}_{011}$ cavity (Billerica, MA). Samples were loaded in $0.15 \mathrm{~mm}$ I.D. thin quartz capillary tubes before inserting into the cylindrical cavity. Spectra were measured with $1 \mathrm{kHz}$ modulation frequency, 1-2 G modulation amplitude, and 150-200 G scan range. Signal-to-noise ratio (SNR) of a spectrum is defined by peak-to-peak intensity vs. 2 x standard deviations of the background. Spectral simulations to obtain motional correlation times were performed using EasySpin software [56] and details of the fitting are given in the Supporting Information.

\subsection{Induction-Mode Thin-Layer Sample Holders}

Several cylindrical Rexolite ${ }^{\circledR}$ (cross-linked polystyrene, C-Lec Plastics, Inc., Philadelphia, PA) sample holders of $50 \mathrm{~mm}$ outer diameter (O.D.) were designed and tested for signal sensitivity (Figure 1). These sample holders occupy the full $50 \mathrm{~mm} \mathrm{I.D.} \mathrm{of} \mathrm{the} \mathrm{corrugated} \mathrm{HE}_{11} / \mathrm{HE}_{12}$ mode waveguide that feedsmicrowaves to/from the magnetic field $\left(\mathrm{B}_{0}\right)$ center, and are supported by a $57 \mathrm{~mm}$ O.D. brass holder positioned at the end of the waveguide. The sample occupies a shallow 
machined cylindrical recess within the Rexolite $\AA$ base, and is protected by a Rexolite $®$ cap that covers the recess (Figure 1); four separate holders were fabricated with sample spaces of varying depth and diameter (Table 1). The Rexolite ${ }^{\circledR}$ caps and bases were polished to be optically transparent in order to reduce surface roughness. A $1 \mathrm{~mm}$ thick aluminum mirror, mounted on a Tufset (Tufnol Composites Ltd., UK) polyurethane support, is positioned immediately underneath the Rexolite ${ }^{\circledR}$ base in order to reflect microwaves back through the sample to the receiver. To first order, the HE waveguide mode can be approximated as a freely propagating, plane-parallel Gaussian beam with incident and reflected wavevectors normal to the plane of the thin sample. Accordingly, the perpendicular distance $(1.005 \mathrm{~mm})$ between the center of the sample and the mirror is designed to be half a wavelength at $94 \mathrm{GHz}$ within the Rexolite® material (refractive index of 1.59), thereby ensuring that the sample sits within the maximum of the oscillating magnetic ( $\mathrm{B}_{1}$-field) component of the microwave radiation, while also minimizing interaction with the electric component (Figure S2 and S3). Likewise, the Rexolite® cap has been machined to a thickness of $1.507 \mathrm{~mm}$ (3/4 of the wavelength) in order to minimize reflections and maximize transmission to the aqueous sample. The holders were fabricated inhouse by the NHMFL machine shop.

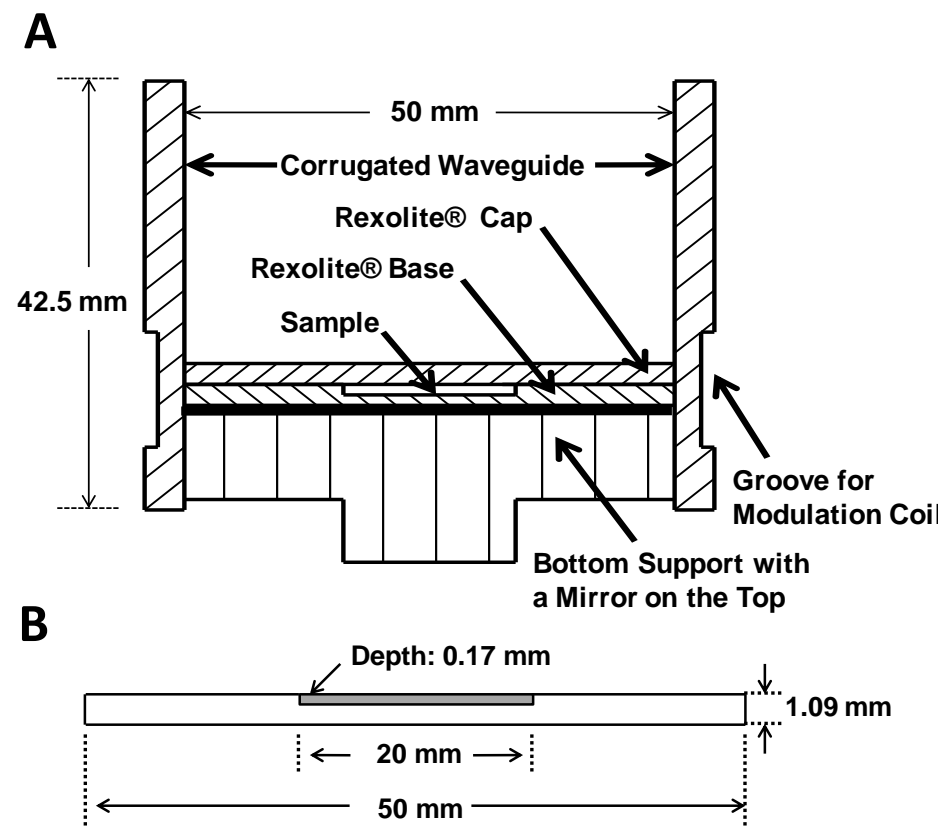

Figure 1. (A) Schematic diagram of the HiPER thin-layer sample holder (See details in section 2.6.). (B) Closer view of the thin-layer recessed sample space of the holder $\mathrm{H} 2$. 
Table 1. Comparison of thin-layer sample holder dimensions and sample volumes.

\begin{tabular}{|l|l|l|l|l|}
\hline Holder Type & \multicolumn{1}{c|}{ H1 } & \multicolumn{1}{c|}{ H2 } & \multicolumn{1}{c|}{ H3 } & \multicolumn{1}{c|}{ H4 } \\
\hline Depth $(\mathrm{mm})$ & $0.5 \pm 0.005$ & $0.17 \pm 0.005$ & $0.17 \pm 0.005$ & $0.085 \pm 0.005$ \\
\hline Diameter $(\mathrm{mm})$ & $40 \pm 0.005$ & $20 \pm 0.005$ & $15 \pm 0.005$ & $20 \pm 0.005$ \\
\hline Volume $(\mu \mathrm{L})$ & $628 \pm 6$ & $53 \pm 2$ & $30 \pm 1$ & $27 \pm 1$ \\
\hline
\end{tabular}

\section{Results}

\subsection{Concentration Sensitivity of Induction-Mode Thin-Layer Sample Holders}

One of the many advantages of the non-resonant quasi-optical approach is a reduced constraint on sample volume in comparison to cavity-based spectrometers (Fabry-Pérot resonators are an exception that we discuss later). Although there is an obvious tradeoff in microwave $B_{1}$-field, one can often compensate for this by increasing the sample volume, thereby achieving overall gains in concentration sensitivity. The plane-wave nature of the HE-mode means that it is only in the direction parallel to microwave propagation that the phases of the $E$ - and $B_{1}$-fields vary over length scales set either by the wavelength (low-loss media) or penetration depth (lossy media). By contrast, these fields vary strongly in at least two dimensions in a cylindrical $\mathrm{TE}_{011}$ cavity; hence the far tighter volume constraint for aqueous (lossy) samples. We therefore set out to examine the sensitivity of four different holders with varying sample dimensions using a series of TEMPO solutions with concentrations in the 2-200 $\mu \mathrm{M}$ range. All spectra in Figure 2A were obtained with $1 \mathrm{G}$ modulation amplitude, a 100 millisecond time constant, over a $150 \mathrm{G}$ sweep range ( 75 seconds per spectrum).

With a view to maximizing concentration sensitivity, a first holder (H1) was designed for a large sample volume having a thickness of $0.5 \mathrm{~mm}$, which is about twice the room temperature penetration depth in water at $94 \mathrm{GHz}(\sim 0.25 \mathrm{~mm})$; likewise, a diameter of $40 \mathrm{~mm}$ was chosen to span most of the waveguide cross section. Representative TEMPO spectra are displayed in Figure 2A, with the SNR determined for each spectrum plotted versus concentration in Figure S4. Although excellent sensitivity is demonstrated with holder H1, its large volume $(628 \mu \mathrm{L})$ presents challenges for many biological studies where the amount of sample is typically limited. The key to obtaining a strong inductive mode EPR signal involves simultaneously maximizing 
the resonant interaction with the magnetic $\left(B_{1}\right)$ component of the microwave field within the sample, whilst minimizing dielectric losses due to the non-resonant interaction with the electric component. Holder $\mathrm{H} 1$ performs well in terms of the former, but not the latter. The resultant decay of the microwave $B_{1}$-field within the relatively thick sample therefore means that it is mostly spins near the top surface that contribute to the reflected cross-polar EPR signal. In other words, much of the volume employed in holder $\mathrm{H} 1$ is redundant.

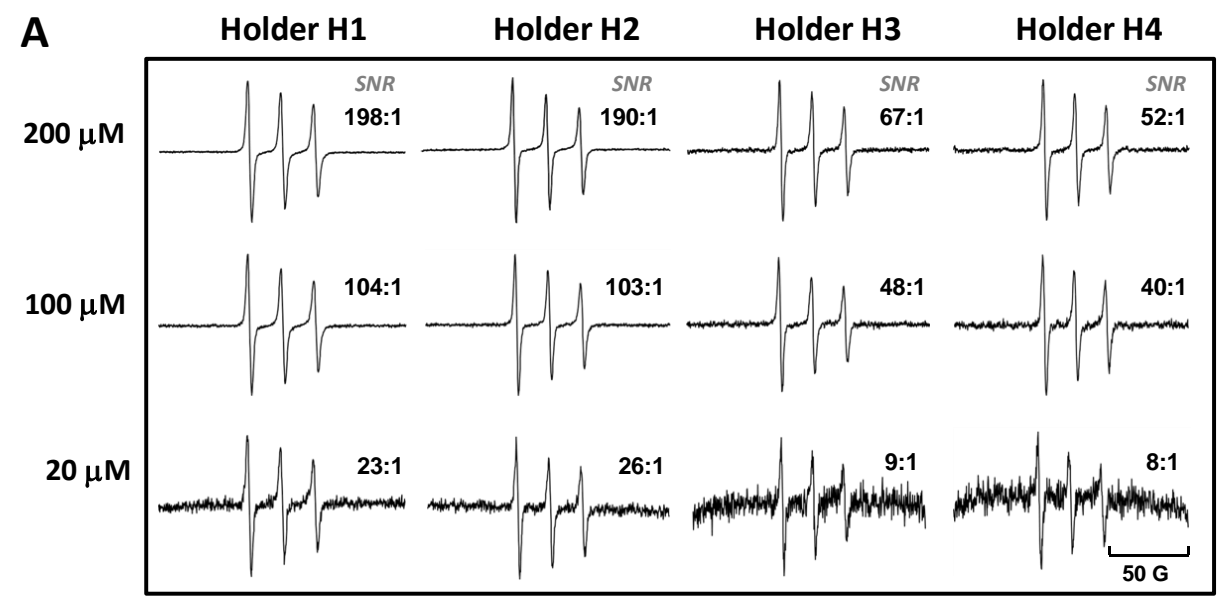

B

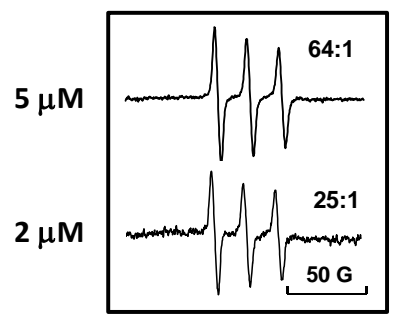

C

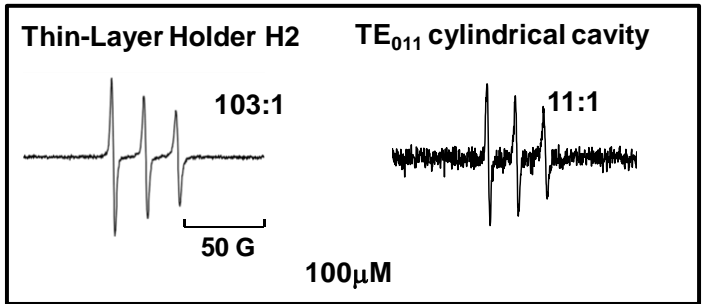

Figure 2. (A) Absorption area normalized EPR spectra of TEMPO solutions using different thinlayer sample holders. Spectra are vertically offset for clarity. Spectra were collected using 150 Gauss sweep width, 1 Gauss modulation-amplitude, 100 millisecond time constant, and 1 average at room temperature (75 second scans). SNRs are marked in the upper-right corners of the spectra. (B) Spectra of $2 \mu \mathrm{M}$ and $5 \mu \mathrm{M}$ TEMPO using sample holder H2. Both spectra were collected using 150 Gauss sweep width, 2 Gauss modulation-amplitude, 1 second time constant, and 6 averages (13 minute scans) at room temperature. (C) Spectra of $100 \mu$ M TEMPO using holder $\mathrm{H} 2$ on HiPER and a $\mathrm{TE}_{011}$ cylindrical cavity on a Bruker E680 spectrometer. Comparable parameters were chosen as follows: (i) HiPER holder H2: 1 Gauss modulation amplitude, 100 millisecond time constant, 100 millisecond conversion time, 150 Gauss sweep width, and one scan (75 seconds); (ii) $\mathrm{TE}_{011}$ cylindrical cavity: 1 Gauss modulation amplitude, 80 millisecond time constant, 80 millisecond conversion time, 150 Gauss sweep width, and one scan (82 seconds). Note: slightly different time constants were used for the two spectrometers because of the different setups of their lock-in amplifiers. 
In light of the above, a second holder $\mathrm{H} 2$ was fabricated of reduced thickness and diameter, in an attempt to minimize the sample volume without significantly compromising sensitivity. Reducing the sample thickness increases the transmitted signal, thereby increasing the importance of the aluminum mirror below the sample (this mirror was almost redundant in the case of $\mathrm{H} 1$ due to strong microwave attenuation within the sample). When positioned correctly (Figure S2 and S3), the resultant standing wave significantly reduces the electric field within the sample, in turn decreasing the dielectric losses. This problem can be optimized in silico on the basis of a transmission line model, using transfer matrices that consider the dielectric properties of all different components/interfaces within the holder, as well as the small permeability changes in the sample on resonance (for one circular polarization). This modeling approach is similar to that described by Earle et al. in [57]. The sample is assumed to have a complex permittivity appropriate to water at $94 \mathrm{GHz}\left(\varepsilon=8.057, \varepsilon^{\prime}=13.66\right)$ [50], and a complex permeability that is different for the two circular polarization states. The model then computes the reflected cross-polar amplitude in which the EPR signal is encoded.

On this basis, a sample thickness of $0.17 \mathrm{~mm}$ was estimated to give the maximum EPR intensity. To be noted, this value is larger than the reported $0.025 \mathrm{~mm}$ aqueous sample thickness at $95 \mathrm{GHz}$ using a Febry-Perot resonator [58]. This is due to the lower finesse (lower Q) of the sample holders used here than the Febry-Perot resonator, which is more sensitive to dielectric losses in the sample and requires smaller optimal sample thickness. Meanwhile, consideration of the approximately Gaussian microwave beam profile transverse to the propagation direction reveals that most of the EPR signal (integral of the $B_{1}$ field over the sample volume) [59] originates from a cylindrical region at the center of the waveguide having a 15-20 $\mathrm{mm}$ diameter (beyond which $B_{1}$ falls below 1/e of its maximum). Consequently, holder $\mathrm{H} 2$ was fabricated with a thickness of $0.17 \mathrm{~mm}$ and a diameter of $20 \mathrm{~mm}$, giving a sample volume of $53 \mu \mathrm{L}$. Remarkably, the SNRs obtained for this holder at each concentration are virtually identical to those of H1 (Figures 2A and S3), demonstrating a dramatic gain in absolute sensitivity and the same concentration sensitivity with $<10 \%$ of the sample volume.

In order to further validate our approach and to assess the consequences of a further reduction in sample volume, two additional holders were fabricated with roughly half the volume of $\mathrm{H} 2$. Here, we wanted to examine the performance of smaller volume holders for cases where biological 
samples were limited. In one holder $(\mathrm{H} 3)$, the diameter was reduced to $15 \mathrm{~mm}$ whilst maintaining the $0.17 \mathrm{~mm}$ thickness, giving a volume of $\sim 30 \mu \mathrm{L}$. Meanwhile, the thickness of holder $\mathrm{H} 4$ was reduced by a factor of two (to $0.085 \mathrm{~mm}$ ), whilst maintaining the same $20 \mathrm{~mm}$ diameter as $\mathrm{H} 2$, giving a volume of $\sim 27 \mu \mathrm{L}$. As can be seen from Figures $2 \mathrm{~A}$ and S3, quite dramatic reductions (more than a factor of two in both cases) in SNRs are observed relative to $\mathrm{H} 2$ at all concentrations. These measurements therefore confirm the results of the transmission line model calculations and demonstrate that holder $\mathrm{H} 2$ provides the optimum compromise between sample volume and concentration sensitivity. The limits of this sensitivity are further demonstrated in Figure 2B, which displays results for lower concentration samples, where $5 \mu \mathrm{M}$ and $2 \mu \mathrm{M}$ TEMPO were utilized with increased signal averaging compared to Figure 2A (see caption). A SNR of 64:1 was achieved for the former, whereas a SNR of 24:1 was obtained for the $2 \mu \mathrm{M}$ sample. Holder $\mathrm{H} 2$ thus provides a convenient geometry and exceptional concentration sensitivity, and was therefore subsequently utilized to assess the overall performance of induction-mode thin-layer sample holders for studies of spin-labeled biological macromolecular samples.

Enhancements in concentration sensitivity relative to the Bruker $\mathrm{TE}_{011}$ cylindrical resonator on a commercial E680 spectrometer were demonstrated by collecting spectra under comparable conditions for $100 \mu \mathrm{M}$ TEMPO (Figure 2C). Results demonstrate a $\sim 9$-fold SNR improvement for sample holder $\mathrm{H} 2$. In other words, significant gains in concentration sensitivity can be achieved for aqueous solution samples by using thin-layer induction mode sample holders.

\subsection{Structural Transitions in Intrinsically Disordered Proteins}

In order to evaluate the sensitivity of the thin-layer sample holder $\mathrm{H} 2$ for SDSL applications of IDPs, EPR spectra of spin-labeled (SL) $\mathrm{IA}_{3}$ were collected. $\mathrm{IA}_{3}$ is a 68 amino acid protein that is unstructured in solution and is a potent inhibitor of yeast aspartic proteinase A (YPRA) [60]. IA 3 undergoes an unstructured to $\alpha$-helical conformation transition upon binding to YPRA, or in the presence of a high content of TFE in the solution [61]. To obtain an EPR signal, residue E10 at the N-termini of $\mathrm{IA}_{3}$ was mutated to cysteine and subsequently labeled with an IAP spin label (P1). Figure 3A shows EPR spectra of SL-IA 3 at spin concentrations varying from $20 \mu \mathrm{M}$ to 200 $\mu \mathrm{M}$, with $0 \%$ and $30 \%$ TFE to represent the unstructured and $\alpha$-helical conformation, respectively. All spectra were collected at $300 \mathrm{~K}$ by averaging 6 scans over a $200 \mathrm{G}$ sweep range 
(13 minutes per scan). The magnetic field modulation amplitude was set to $2 \mathrm{G}$, and a lock-in amplifier with a time constant of 1 second was used in the demodulation of the EPR resonant signal.
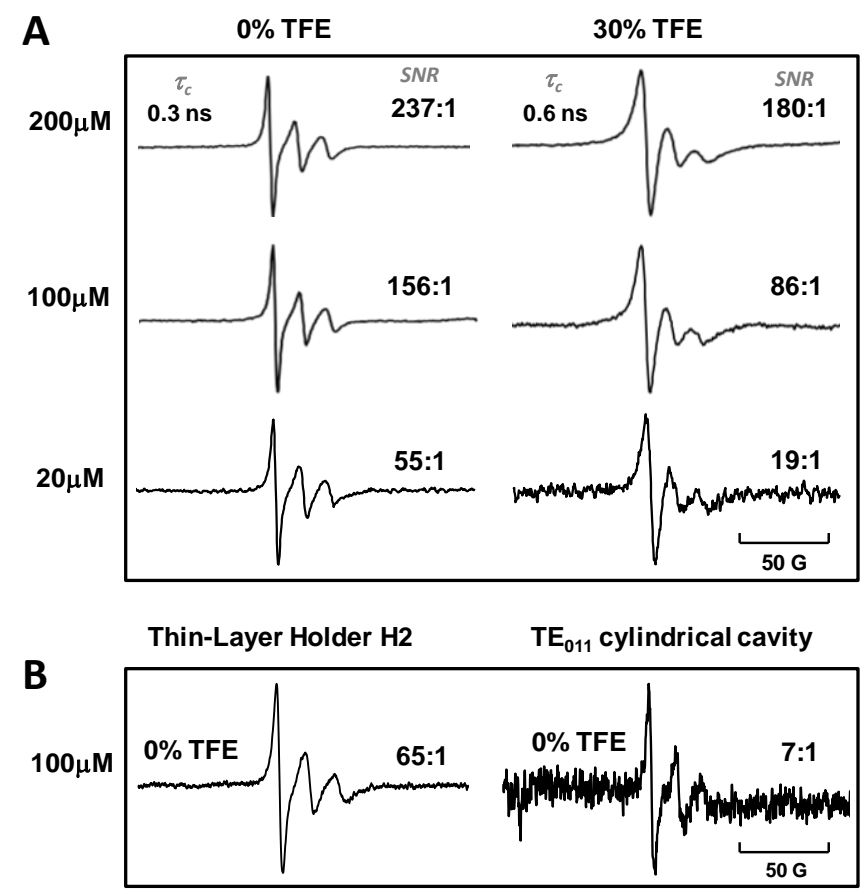

Figure 3. Intensity normalized W-band spectra of $\mathrm{SL}_{-} \mathrm{IA}_{3}$ at site E10P1. (A) Spectra are vertically offset showing how values of the SNR change as a function of concentration with $0 \%$ and $30 \%$ TFE at $300 \mathrm{~K}$. Spectra were collected using sample holder H2 with $200 \mathrm{G}$ sweep width, $2 \mathrm{G}$ modulation-amplitude, 1 second time constant and 6 averages (13 minute scans). The spectral line shape and rotational correlation time changes for the $0 \%$ and $30 \%$ TFE states are consistent with an unstructured to helical transition (simulated spectra are given in Supporting Information). (B) Comparison of concentration sensitivity of the $\mathrm{H} 2$ holder on HiPER and a $\mathrm{TE}_{011}$ cylindrical cavity on a Bruker E680 spectrometer. Spectra are acquired for a $100 \mu \mathrm{M}$ sample. Comparable spectral parameters were utilized as follows: (1) HiPER holder H2: 2 G modulation amplitude, 1 second time constant, 1 second conversion time, $200 \mathrm{G}$ sweep width, and one scan (17 minutes). $\mathrm{TE}_{011}$ cylindrical cavity: $2 \mathrm{G}$ modulation amplitude, 1.3 second time constant, 1.3 second conversion time, $200 \mathrm{G}$ sweep width, and one scan (22 minutes). Note: slightly different time constants were used for the two spectrometers because of the different setups of their lock-in amplifiers.

For SL-IA 3 concentrations in the 100 to $200 \mu \mathrm{M}$ range in $0 \%$ and $30 \%$ TFE, SNR values ranging from 90:1 to 240:1 were obtained. Even for concentrations as low as $20 \mu \mathrm{M}$ in $30 \%$ TFE, SNR values of 19:1 or higher were achieved (Figures $3 \mathrm{~A}$ and S5). In the 30\% TFE solution, $\mathrm{IA}_{3}$ becomes helical, and due to the ordered helical backbone restricting the spin-label motion an increase in the spectral correlation time increases from $0.3 \mathrm{~ns}$ in the unfolded state to $0.6 \mathrm{~ns}$ in 
the folded state is observed. Corresponding spectral simulations are included in Figure S7, where the slower motion is reflected in a broadening of the spectra, consistent with our previous Xband investigations [37, 43]. This spectral broadening impacts overall signal and is reflected in reduced SNR in the 30\% TFE samples compared to the 0\% TFE samples (Figure 3A). Again, a 9-fold enhancement in concentration sensitivity for sample holder $\mathrm{H} 2$ relative to the Bruker $\mathrm{TE}_{011}$ cylindrical resonator on a commercial E680 spectrometer was demonstrated for $100 \mu \mathrm{M} 0 \%$ TFE SL-IA 3 (Figures 3B).

\subsection{Characterizing Conformational Dynamics in Large RNAs}

There is growing interest in characterizing conformational changes in large RNAs and, as SDSL has matured for characterizing RNAs, it is clear that oftentimes the resultant X-band spectra reveal relatively fast motion, with correlation times ranging from 0.3 to $3 \mathrm{~ns}$. Meanwhile, small RNA molecules have been found to tumble in the fast-motion time regime [9, 53, 62]. Recent studies have used SDSL to elucidate structure and dynamics of RNAs in a number of cellular pathways [9, 53, 62]. RNAs are notorious for difficulties in sample preparations, including instability and low-yield. Regarding the study of large dynamic RNAs such as riboswitches, local regions may undergo conformational changes with local dynamics within this fast motional regime. Our previous R5 spin labeling studies of the kink-turn motif in the glycine riboswitch (Figure 4A) is one such example [54].

For preparing spin labeled (SL) glycine riboswitch, we utilize a splinted ligation method that routinely generates approximately $360 \mu \mathrm{g}$ of purified SL-RNA. For EPR investigations, this equates to $30 \mu \mathrm{L}$ of $150 \mu \mathrm{M}$ total sample from each round of ligation; hence, the optimized concentration sensitivity of the $\mathrm{H} 2$ sample holder is essential for these investigations given that concentrating the sample to smaller volumes may result in non-specific aggregation. Additionally, radical reduction is oftentimes a challenge when labeling RNA bases. We typically observe < $40 \%$ spin concentration for a given thiouridyl base labeling; thus further necessitating the best concentration sensitivity. Figure 4 shows W-band data obtained with sample holder H2 for $53 \mu \mathrm{L}$ of $30 \mu \mathrm{M}$ (spin concentration) $232 \mathrm{nt} V C$ glycine riboswitch containing an IAP modified thiouridyl base (U2) in the kink-turn region (Figure 4B). When in water only, RNA is 
expected to be unstructured, and W-band spectrum (Figure 4C top and S8) with a SNR value of 18:1 reveal disorder, with spectral simulation giving a correlation time of $0.4 \mathrm{~ns}$. It has previously been shown that, upon addition of salts and ligands, the $V C$ glycine riboswitch folds with formation of the kink turn $[53,54,63,64]$ where, based upon comparisons to X-ray models of other k-turn motifs [65, 66], the modified U2-base is expected to become base paired/base stacked. Hence, a decrease in dynamics (an increase in correlation time) is anticipated. Indeed, markedly broadened spectra are obtained (Figure 4C bottom and S8, S9) in solution conditions that promote folding. The data for the folded state has a SNR value of 9:1 and spectral simulations indicate an increased correlation time of $1.0 \mathrm{~ns}$. Given that the above experiments (Figs 2C and 3B) demonstrated a 9-10 x improvement in the of concentration sensitivity of the H2 sample holder on HiPER compared to our commercial Bruker $\mathrm{TE}_{011}$ cylindrical resonator on a E680 spectrometer; these data would likely be unobtainable on our commercial system.

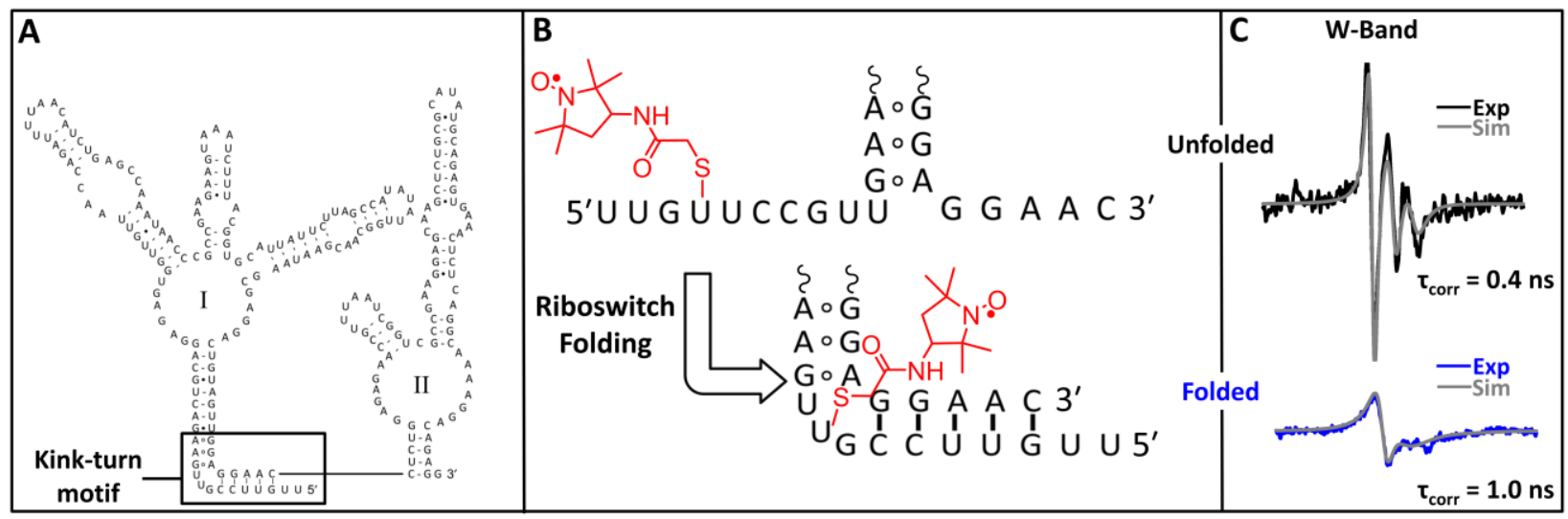

Figure 4. (A) Secondary structure of the 232 nt $V C$ glycine riboswitch with the kink-turn motif boxed in black. (B) Enlarged kink-turn motif structure depicted in the unfolded (top) and folded (bottom) states showing IAP on the chosen modified thiouridyl base. (C) Experimental and simulated (grey) W-Band spectra for the unfolded (top, black) and folded (bottom, blue) riboswitch states with signal to noise ratios for each shown. The arrow indicates features in the folded state spectrum that may arise from a small fraction of unfolded RNA $(<1 \%)$ Spectra were collected using $200 \mathrm{G}$ sweep width, 1 second time constant, $4 \mathrm{G}$ modulation amplitude, and 5 (unfolded) and 6 (folded) averages. 


\section{Discussion}

\subsection{Sensitivity of HiPER Thin-Layer Sample Holders}

Despite the many appealing potential applications involving biological macromolecules, the development of high-field SDSL EPR has lagged behind its more popular low field X-band counterpart. This is due to the many technical challenges associated with the detection of EPR signals from aqueous samples at $\mathrm{W}$-band $(94 \mathrm{GHz})$ and above. To design a sample holder at these high frequencies, the most common practice is to use a resonator [50, 58, 67]. All other things being equal, one would expect significantly enhanced sensitivity at higher fields and frequencies on account of the increased sample magnetization $\left(\propto B_{0} / T\right)$. However, the reduction in wavelength typically results in smaller resonators and, hence, smaller sample sizes. The decreased active volume, coupled with the reduced penetration into aqueous samples and the many technical challenges associated with the design of EPR spectrometers operating at high microwave frequencies, usually results in an overall reduction in concentration sensitivity when compared to X-band. In particular, sample volumes are typically reduced to sub-microliter levels at W-band and above. For instance, the commercial W-band Bruker $\mathrm{TE}_{011}$ cylindrical cavity has a maximum sample volume of $\sim 0.1 \mu \mathrm{L}$, while a typical Fabry-Pérot resonator has an effective volume of $\sim 0.5 \mu \mathrm{L}$ for aqueous samples [58]. Although these resonators offer superior absolute spin sensitivity, their concentration sensitivities are much lower than at $\mathrm{X}$-band due to dramatically reduced sample volumes. Consequently, the increased concentration requirement abrogates effectiveness in terms of studies aimed at biological macromolecules such as IDPs and RNAs, given that these molecules are oftentimes hard to concentrate above 20-100 $\mu \mathrm{M}$.

It is for the aforementioned reasons that we have been motivated to build a sample holder that is suitable for studying spin-labeled samples under conditions and concentrations that are comparable to the more traditional X-band studies. We recently demonstrated the use of W-band frequencies for investigations for IDPs [43]. However, we also showed that adequate SNRs are only obtained for $\mathrm{IA}_{3}$ at sample concentrations of order $1 \mathrm{mM}$, which interfered with our attempts to properly determine TFE concentrations [68]. Here, we have shown that concentration sensitivities in the 10-100 $\mu \mathrm{M}$ range can be achieved using non-resonant holders that are suitable for $10-100 \mu \mathrm{L}$ sample volumes. Because both the sample volume and $\mathrm{B}_{1}$ field strength contribute 
to concentration sensitivity, a large volume can compensate for a relatively low $\mathrm{B}_{1}$ field in comparison to a resonator operating at the same microwave power level. Moreover, the maximum $B_{1}$ field is often limited in cases where spin saturation effects come into play. In such situations, a low-B $\mathrm{B}_{1}$ field, large volume non-resonant sample holder is actually preferable for $\mathrm{CW}$ EPR studies.

We conclude this section by considering possibilities for sample volume reduction, whilst maintaining or further increasing concentration sensitivity. This obviously requires increases in $\mathrm{B}_{1}$ field. The most straightforward approach will be to increase the source power; recent advances in W-band technology make this a realistic possibility. Another approach involves reducing the waveguide/ beam diameter. This would result in offsetting effects due to an increase in $\mathrm{B}_{1}$ and a reduction in the diameter/volume of the useful sample space. Thus, concentration sensitivity would not be enhanced. However, this would provide an advantage in terms of reduced sample volume. The final strategy involves the design of a Fabry-Pérot resonator with a beam waist of comparable diameter to the present sample holder (H2). Although technically challenging, this would almost certainly provide opportunities for increasing concentration sensitivity and/or reducing the sample volume.

\subsection{Idealized Sample Sizes for SDSL}

Our overarching goal for this investigation was to generate sample holders for CW EPR investigations targeted at characterizing conformational dynamics in biological systems such as IDPs and RNAs, with extensions to studies of membranes and membrane proteins at $94 \mathrm{GHz}$. The results demonstrate the relationships between geometry variables (depth and diameter) and concentration sensitivity of the thin cylindrically shaped aqueous films, and that these parameters are ultimately dictated by the wavelength of the incident microwave radiation and the permittivity of water at $94 \mathrm{GHz}$. The results further demonstrate the feasibility and facile manner of sample loading and sample sizes utilized; typically $30-50 \mu \mathrm{L}$ of $20-50 \mu \mathrm{M}$ concentration is required for publication quality data that can be collected within minutes to hours for SDSL investigations of motion in the sub-ns to $3 \mathrm{~ns}$ regime. This improvement in concentration sensitivity over the commercial Bruker spectrometer is essential when studying systems that are 
sample limited, concentration limited, or where investigators want to interrogate molecular interactions under conditions that mimic concentrations found within cells. It is also noteworthy that the geometric shape of these sample holders should be much more amenable to in-cell EPR investigations, where small capillary diameters can induce "stress-like" conditions for cells.

\subsection{Application in Multi-Frequency SDSL}

This study highlights the suitability of utilizing thin-layer sample holders in studies aimed at characterizing molecular dynamics and structural transitions of biological macromolecules at high fields. For instance, CW spectra at W- and D- bands provide an enhanced capability to detect sub-nanosecond correlation times [43]. This is particularly suitable for studying IDPs, in which the time scale normally falls into the 0.1 to 2 ns region [37, 38, 44-48, 69-72]. Furthermore, EPR analyses at multiple frequencies allow one to unravel complex dynamics including distinct motional timescales [34-36]. Multi-frequency EPR allows one to combine the characterization of dynamics in various motional regimes that are typical for biological systems: fast motion detected by very high frequencies and slow motion using lower frequencies. A study of phospholamban (PLB) illustrates this point, where global analysis of spectra at two frequencies yielded values for rotational correlation times and order parameters that were much more precisely determined than at either frequency alone [34]. Other studies have also demonstrated that multi-frequency EPR is an appealing approach to circumvent the low resolution at X-band for sub-nanosecond time scales [29, 31, 36, 73].

However, the low concentration sensitivities of currently available technologies and instruments at high-fields have largely prevented the investigation of low-yield biological samples, including IDPs. For instance, spin concentrations of a few hundred $\mu \mathrm{M}$ to $1 \mathrm{mM}$ are usually needed to obtain satisfactory $\mathrm{CW}$ spectra and SNR values for nitroxide-labeled proteins using a commercial cylindrical resonator at $94 \mathrm{GHz}$. This level of concentration is not always feasible for biological samples for which protein aggregation is a serious concern at such high concentrations. The present study demonstrates that W-band investigations at much lower concentrations are feasible. 


\subsection{Applications to Other Biological Samples}

Other applications of high-field and multi-frequency EPR will also benefit from the concentration sensitivity of HiPER thin-layer sample holders, such as the study of membrane biophysics, membrane-protein interactions and membrane proteins. High fields provide increased sensitivity to measure the structural parameters of membranes and membraneassociated proteins, such as fluidity, polarity, orientation and membrane order [26, 27, 30, 31, 74, 75]. An improved sensitivity of sample holders at high fields will greatly facilitate the unraveling of these complex parameters.

\section{Conclusions}

The present study examined the concentration sensitivity of thin-layer cylindrical sample holders for induction-mode CW EPR studies of aqueous solution samples at $94 \mathrm{GHz}$, with application to SDSL biomolecules. The optimum sample holder has a $53 \mu \mathrm{L}$ volume in a thin-layer geometry with a large surface area. The feasibility of utilizing these sample holders for biological applications using the HiPER spectrometer at the NHMFL was demonstrated by inducing structural transitions and monitoring concomitant spectral line shape changes of an intrinsically disordered protein $\left(\mathrm{IA}_{3}\right)$ and base stacking that occurs during k-turn formation of a large RNA. A concentration sensitivity of $2 \mu \mathrm{M}$ was achieved for a TEMPO standard and 20-30 $\mu \mathrm{M}$ for the biological samples. The results of this study illustrate the potential application for characterizing dynamic spin-labeled biological systems, benefiting from the concentration sensitivity of thinlayer sample holders and enhanced spectral resolution of fast motions at high magnetic fields. These applications may be extended to high-field and multi-frequency EPR studies of other biological samples.

\section{Acknowledgments}

This work was performed at the National High Magnetic Field Laboratory (NHMFL), which is supported by the National Science Foundation (DMR-1157490) and the State of Florida. L.S. acknowledges support from the National Institutes of Health (AI091693) and the NHMFL User 
Collaboration Grants Program (Award No. 5080). G.E.F. acknowledges support from the National Science Foundation (MCB-1329467) and the National Institutes of Health (GM105409 and S10RR031603). S.H. acknowledges support from the National Science Foundation (DMR1309463). J.M.E acknowledges support from the National Science Foundation (DGE-0802270). 


\section{References}

[1] C. Altenbach, D. A. Greenhalgh, H. G. Khorana, and W. L. Hubbell. A collision gradient method to determine the immersion depth of nitroxides in lipid bilayers: application to spin-labeled mutants of bacteriorhodopsin, Proc Natl Acad Sci U S A. 91 (1994) 1667-1671.

[2] I. D. Sahu, R. M. McCarrick, K. R. Troxel, R. Zhang, H. J. Smith, M. M. Dunagan, M. S. Swartz, P. V. Rajan, B. M. Kroncke, C. R. Sanders, and G. A. Lorigan. DEER EPR measurements for membrane protein structures via bifunctional spin labels and lipodisq nanoparticles, Biochemistry. 52 (2013) 6627-6632.

[3] D. S. Cafiso. Identifying and Quantitating Conformational Exchange in Membrane Proteins Using Site-Directed Spin Labeling, Accounts Chem Res. 47 (2014) 3102-3109.

[4] A. K. Smith, and J. H. Freed. Dynamics and ordering of lipid spin-labels along the coexistence curve of two membrane phases: an ESR study, Chem Phys Lipids. 165 (2012) 348-361.

[5] H. Raghuraman, S. M. Islam, S. Mukherjee, B. Roux, and E. Perozo. Dynamics transitions at the outer vestibule of the KcsA potassium channel during gating, Proc Natl Acad Sci U S A. 111 (2014) 1831-1836.

[6] K. Kazmier, S. Sharma, S. M. Islam, B. Roux, and H. S. McHaourab. Conformational cycle and ioncoupling mechanism of the $\mathrm{Na}+$ /hydantoin transporter Mhp1, Proc Natl Acad Sci U S A. 111 (2014) 14752-14757.

[7] B. Vileno, J. Chamoun, H. Liang, P. Brewer, B. D. Haldeman, K. C. Facemyer, B. Salzameda, L. Song, H. C. Li, C. R. Cremo, and P. G. Fajer. Broad disorder and the allosteric mechanism of myosin II regulation by phosphorylation, Proc Natl Acad Sci U S A. 108 (2011) 8218-8223.

[8] B. P. Binder, S. Cornea, A. R. Thompson, R. J. Moen, and D. D. Thomas. High-resolution helix orientation in actin-bound myosin determined with a bifunctional spin label, Proc Natl Acad Sci U S A. 112 (2015) 7972-7977.

[9] P. Z. Qin, K. Hideg, J. Feigon, and W. L. Hubbell. Monitoring RNA base structure and dynamics using site-directed spin labeling, Biochemistry. 42 (2003) 6772-6783.

[10] V. Meyer, M. A. Swanson, L. J. Clouston, P. J. Boratynski, R. A. Stein, H. S. McHaourab, A. Rajca, S. S. Eaton, and G. R. Eaton. Room-temperature distance measurements of immobilized spinlabeled protein by DEER/PELDOR, Biophysical Journal. 108 (2015) 1213-1219.

[11] G. E. Fanucci, and D. S. Cafiso. Recent advances and applications of site-directed spin labeling, Curr Opin Struct Biol. 16 (2006) 644-653.

[12] L. Song, Z. Y. Sun, K. E. Coleman, M. B. Zwick, J. S. Gach, J. H. Wang, E. L. Reinherz, G. Wagner, and M. Kim. Broadly neutralizing anti-HIV-1 antibodies disrupt a hinge-related function of gp41 at the membrane interface, Proc Natl Acad Sci U S A. 106 (2009) 9057-9062.

[13] L. D. Jennings-Antipov, L. Song, and R. J. Collier. Interactions of anthrax lethal factor with protective antigen defined by site-directed spin labeling, Proc Natl Acad Sci U S A. 108 (2011) 1868-1873.

[14] M. Kim, L. Song, J. Moon, Z. Y. Sun, A. Bershteyn, M. Hanson, D. Cain, S. Goka, G. Kelsoe, G. Wagner, D. Irvine, and E. L. Reinherz. Immunogenicity of membrane-bound HIV-1 gp41 membrane-proximal external region (MPER) segments is dominated by residue accessibility and modulated by stereochemistry, J Biol Chem. 288 (2013) 31888-31901.

[15] C. Altenbach, S. L. Flitsch, H. G. Khorana, and W. L. Hubbell. Structural studies on transmembrane proteins. 2. Spin labeling of bacteriorhodopsin mutants at unique cysteines, Biochemistry. 28 (1989) 7806-7812.

[16] G. Jeschke. Conformational dynamics and distribution of nitroxide spin labels, Prog Nucl Mag Res Sp. 72 (2013) 42-60. 
[17] I. M. de Vera, M. E. Blackburn, L. Galiano, and G. E. Fanucci. Pulsed EPR distance measurements in soluble proteins by site-directed spin labeling (SDSL), Curr Protoc Protein Sci. 74 (2013) Unit 1717.

[18] L. Columbus, and W. L. Hubbell. A new spin on protein dynamics, Trends Biochem Sci. 27 (2002) 288-295.

[19] X. J. Zhang, P. Cekan, S. T. Sigurdsson, and P. Z. Qin. Studying Rna Using Site-Directed SpinLabeling and Continuous-Wave Electron Paramagnetic Resonance Spectroscopy, Methods in Enzymology, Vol 469: Biophysical, Chemical, and Functional Probes of Rna Structure, Interactions and Folding, Pt B. 469 (2009) 303-328.

[20] P. Devaux, and H. M. McConnell. Lateral diffusion in spin-labeled phosphatidylcholine multilayers, J Am Chem Soc. 94 (1972) 4475-4481.

[21] W. L. Hubbell, C. J. Lopez, C. Altenbach, and Z. Y. Yang. Technological advances in site-directed spin labeling of proteins, Curr Opin Struc Biol. 23 (2013) 725-733.

[22] I. M. C. van Amsterdam, M. Ubbink, G. W. Canters, and M. Huber. Measurement of a Culecu Distance of $26 \AA$ by a Pulsed EPR Method, Angewandte Chemie International Edition. 42 (2003) 62-64.

[23] J. Voss, L. Salwinski, H. R. Kaback, and W. L. Hubbell. A method for distance determination in proteins using a designed metal ion binding site and site-directed spin labeling: Evaluation with T4 lysozyme, P Natl Acad Sci USA. 92 (1995) 12295-12299.

[24] A. Potapov, H. Yagi, T. Huber, S. Jergic, N. E. Dixon, G. Otting, and D. Goldfarb. Nanometer-Scale Distance Measurements in Proteins Using Gd3+ Spin Labeling, J Am Chem Soc. 132 (2010) 90409048.

[25] D. T. Edwards, T. Huber, S. Hussain, K. M. Stone, M. Kinnebrew, I. Kaminker, E. Matalon, M. S. Sherwin, D. Goldfarb, and S. I. Han. Determining the Oligomeric Structure of Proteorhodopsin by Gd3+-Based Pulsed Dipolar Spectroscopy of Multiple Distances, Structure. 22 (2014) 1677-1686.

[26] D. Marsh, D. Kurad, and V. A. Livshits. High-field spin-label EPR of lipid membranes, Magn Reson Chem. 43 Spec no. (2005) S20-25.

[27] J. P. Barnes, and J. H. Freed. Dynamics and ordering in mixed model membranes of dimyristoylphosphatidylcholine and dimyristoylphosphatidylserine: a 250-GHz electron spin resonance study using cholestane, Biophys J. 75 (1998) 2532-2546.

[28] B. Dzikovski, D. Tipikin, and J. Freed. Conformational distributions and hydrogen bonding in gel and frozen lipid bilayers: a high frequency spin-label ESR study, J Phys Chem B. 116 (2012) 66946706.

[29] Z. C. Liang, and J. H. Freed. An assessment of the applicability of multifrequency ESR to study the complex dynamics of biomolecules, Journal of Physical Chemistry B. 103 (1999) 6384-6396.

[30] E. Conte, F. M. Megli, H. Khandelia, G. Jeschke, and E. Bordignon. Lipid peroxidation and water penetration in lipid bilayers: A W-band EPR study, Bba-Biomembranes. 1828 (2013) 510-517.

[31] H. J. Steinhoff, A. Savitsky, C. Wegener, M. Pfeiffer, M. Plato, and K. Mobius. High-field EPR studies of the structure and conformational changes of site-directed spin labeled bacteriorhodopsin, Bba-Bioenergetics. 1457 (2000) 253-262.

[32] L. Mainali, J. S. Hyde, and W. K. Subczynski. Using spin-label W-band EPR to study membrane fluidity profiles in samples of small volume, J Magn Reson. 226 (2013) 35-44.

[33] D. T. Edwards, Z. D. Ma, T. J. Meade, D. Goldfarb, S. G. Han, and M. S. Sherwin. Extending the distance range accessed with continuous wave EPR with Gd3+ spin probes at high magnetic fields, Phys Chem Chem Phys. 15 (2013) 11313-11326.

[34] Y. E. Nesmelov, C. B. Karim, L. Song, P. G. Fajer, and D. D. Thomas. Rotational dynamics of phospholamban determined by multifrequency electron paramagnetic resonance, Biophys J. 93 (2007) 2805-2812. 
[35] Z. W. Zhang, M. R. Fleissner, D. S. Tipikin, Z. C. Liang, J. K. Moscicki, K. A. Earle, W. L. Hubbell, and J. H. Freed. Multifrequency Electron Spin Resonance Study of the Dynamics of Spin Labeled T4 Lysozyme, Journal of Physical Chemistry B. 114 (2010) 5503-5521.

[36] Z. C. Liang, Y. Lou, J. H. Freed, L. Columbus, and W. L. Hubbell. A multifrequency electron spin resonance study of T4 lysozyme dynamics using the slowly relaxing local structure model, Journal of Physical Chemistry B. 108 (2004) 17649-17659.

[37] N. L. Pirman, E. Milshteyn, L. Galiano, J. C. Hewlett, and G. E. Fanucci. Characterization of the disordered-to-alpha-helical transition of IA(3) by SDSL-EPR spectroscopy, Protein Sci. 20 (2011) 150-159.

[38] B. Morin, J. M. Bourhis, V. Belle, M. Woudstra, F. Carriere, B. Guigliarelli, A. Fournel, and S. Longhi. Assessing induced folding of an intrinsically disordered protein by site-directed spinlabeling electron paramagnetic resonance spectroscopy, J Phys Chem B. 110 (2006) 2059620608.

[39] P. Z. Qin, J. Feigon, and W. L. Hubbell. Site-directed spin labeling studies reveal solution conformational changes in a GAAA tetraloop receptor upon $\mathrm{Mg}(2+)$-dependent docking of a GAAA tetraloop, J Mol Biol. 351 (2005) 1-8.

[40] M. Martinho, J. Habchi, Z. El Habre, L. Nesme, B. Guigliarelli, V. Belle, and S. Longhi. Assessing induced folding within the intrinsically disordered C-terminal domain of the Henipavirus nucleoproteins by site-directed spin labeling EPR spectroscopy, J Biomol Struct Dyn. 31 (2013) 453-471.

[41] E. Mileo, E. Etienne, M. Martinho, R. Lebrun, V. Roubaud, P. Tordo, B. Gontero, B. Guigliarelli, S. R. A. Marque, and V. Belle. Enlarging the Panoply of Site-Directed Spin Labeling Electron Paramagnetic Resonance (SDSL-EPR): Sensitive and Selective Spin-Labeling of Tyrosine Using an Isoindoline-Based Nitroxide, Bioconjugate Chem. 24 (2013) 1110-1117.

[42] M. N. Oda, T. M. Forte, R. O. Ryan, and J. C. Voss. The C-terminal domain of apolipoprotein A-I contains a lipid-sensitive conformational trigger, Nat Struct Biol. 10 (2003) 455-460.

[43] T. M. Casey, Z. Liu, J. M. Esquiaqui, N. L. Pirman, E. Milshteyn, and G. E. Fanucci. Continuous wave W- and D-band EPR spectroscopy offer "sweet-spots" for characterizing conformational changes and dynamics in intrinsically disordered proteins, Biochem Biophys Res Commun. 450 (2014) 723-728.

[44] V. N. Uversky. The multifaceted roles of intrinsic disorder in protein complexes, FEBS Lett. (2015).

[45] H. J. Dyson, and P. E. Wright. Intrinsically unstructured proteins and their functions, Nat Rev Mol Cell Biol. 6 (2005) 197-208.

[46] P. E. Wright, and H. J. Dyson. Intrinsically unstructured proteins: re-assessing the protein structure-function paradigm, J Mol Biol. 293 (1999) 321-331.

[47] B. He, K. Wang, Y. Liu, B. Xue, V. N. Uversky, and A. K. Dunker. Predicting intrinsic disorder in proteins: an overview, Cell Res. 19 (2009) 929-949.

[48] V. N. Uversky. Natively unfolded proteins: a point where biology waits for physics, Protein Sci. 11 (2002) 739-756.

[49] A. K. Dunker, I. Silman, V. N. Uversky, and J. L. Sussman. Function and structure of inherently disordered proteins, Curr Opin Struct Biol. 18 (2008) 756-764.

[50] J. P. Barnes, and J. H. Freed. Aqueous sample holders for high-frequency electron spin resonance, Rev Sci Instrum. 68 (1997) 2838-2846.

[51] G. W. Reginsson, R. I. Hunter, P. A. S. Cruickshank, D. R. Bolton, S. T. Sigurdsson, G. M. Smith, and O. Schiemann. W-band PELDOR with $1 \mathrm{~kW}$ microwave power: Molecular geometry, flexibility and exchange coupling, Journal of Magnetic Resonance. 216 (2012) 175-182.

[52] P. A. Cruickshank, D. R. Bolton, D. A. Robertson, R. I. Hunter, R. J. Wylde, and G. M. Smith. A kilowatt pulsed $94 \mathrm{GHz}$ electron paramagnetic resonance spectrometer with high concentration 
sensitivity, high instantaneous bandwidth, and low dead time, Rev Sci Instrum. 80 (2009) 103102.

[53] J. M. Esquiaqui, G. E. Fanucci, and J. D. Ye. Studying Dynamics and Conformational Changes in the Glycine Riboswitch using Electron Paramagnetic Resonance Spectroscopy, Biophysical Journal. 106 (2014) 281a-281a.

[54] J. M. Esquiaqui, E. M. Sherman, S. A. Ionescu, J. D. Ye, and G. E. Fanucci. Characterizing the Dynamics of the Leader-Linker Interaction in the Glycine Riboswitch with Site-Directed Spin Labeling, Biochemistry. 53 (2014) 3526-3528.

[55] T. E. Edwards, and S. T. Sigurdsson. Modified RNAs as Tools in RNA Biochemistry, Handbook of Rna Biochemistry, Vols 1 and 2, 2nd Edition. (2014) 151-171.

[56] S. Stoll, and A. Schweiger. EasySpin, a comprehensive software package for spectral simulation and analysis in EPR, Journal of Magnetic Resonance. 178 (2006) 42-55.

[57] K. A. Earle, R. Zeng, and D. E. Budil. Transfer matrix method for optimizing quasioptical EPR cavities, Applied Magnetic Resonance. 21 (2001) 275-286.

[58] W. Hofbauer, K. A. Earle, C. R. Dunnam, J. K. Moscicki, and J. H. Freed. High-power $95 \mathrm{GHz}$ pulsed electron spin resonance spectrometer, Rev Sci Instrum. 75 (2004) 1194-1208.

[59] J. E. McKay, D. A. Robertson, P. A. S. Cruickshank, R. I. Hunter, D. R. Bolton, R. J. Wylde, and G. M. Smith. Compact Wideband Corrugated Feedhorns With Ultra-Low Sidelobes for Very High Performance Antennas and Quasi-Optical Systems, leee T Antenn Propag. 61 (2013) 1714-1721.

[60] L. H. Phylip, W. E. Lees, B. G. Brownsey, D. Bur, B. M. Dunn, J. R. Winther, A. Gustchina, M. Li, T. Copeland, A. Wlodawer, and J. Kay. The potency and specificity of the interaction between the IA3 inhibitor and its target aspartic proteinase from Saccharomyces cerevisiae, J Biol Chem. 276 (2001) 2023-2030.

[61] R. Narayanan, O. K. Ganesh, A. S. Edison, and S. J. Hagen. Kinetics of folding and binding of an intrinsically disordered protein: the inhibitor of yeast aspartic proteinase YPrA, J Am Chem Soc. 130 (2008) 11477-11485.

[62] P. Z. Qin, J. Iseri, and A. Oki. A model system for investigating lineshape/structure correlations in RNA site-directed spin labeling, Biochemical and Biophysical Research Communications. 343 (2006) 117-124.

[63] E. M. Sherman, J. Esquiaqui, G. Elsayed, and J. D. Ye. An energetically beneficial leader-linker interaction abolishes ligand-binding cooperativity in glycine riboswitches, RNA. 18 (2012) 496507.

[64] N. J. Baird, and A. R. Ferre-D'Amare. Modulation of quaternary structure and enhancement of ligand binding by the K-turn of tandem glycine riboswitches, RNA. 19 (2013) 167-176.

[65] D. M. Lilley. The structure and folding of kink turns in RNA, Wiley interdisciplinary reviews. RNA. 3 (2012) 797-805.

[66] D. M. Lilley. The K-turn motif in riboswitches and other RNA species, Biochimica et biophysica acta. 1839 (2014) 995-1004.

[67] J. van Tol, L. C. Brunel, and R. J. Wylde. A quasioptical transient electron spin resonance spectrometer operating at 120 and $240 \mathrm{GHzL}$, Rev Sci Instrum. 76 (2005).

[68] N. L. Priman. A multi-frequency electron paramagnetic resonance spectroscopy study of the intrinsically disordered protein, IA3 Disssertation, University of Florida. (2011).

[69] N. Le Breton, M. Martinho, E. Mileo, E. Etienne, G. Gerbaud, B. Guigliarelli, and V. Belle. Exploring intrinsically disordered proteins using site-directed spin labeling electron paramagnetic resonance spectroscopy, Front Mol Biosci. 2 (2015) 21.

[70] V. Belle, S. Rouger, S. Costanzo, E. Liquiere, J. Strancar, B. Guigliarelli, A. Fournel, and S. Longhi. Mapping alpha-helical induced folding within the intrinsically disordered C-terminal domain of 
the measles virus nucleoprotein by site-directed spin-labeling EPR spectroscopy, Proteins. 73 (2008) 973-988.

[71] L. Baronti, J. Erales, J. Habchi, I. C. Felli, R. Pierattelli, and S. Longhi. Dynamics of the intrinsically disordered C-terminal domain of the nipah virus nucleoprotein and interaction with the $x$ domain of the phosphoprotein as unveiled by NMR spectroscopy, Chembiochem. 16 (2015) 268-276.

[72] E. Mileo, M. Lorenzi, J. Erales, S. Lignon, C. Puppo, N. Le Breton, E. Etienne, S. R. Marque, B. Guigliarelli, B. Gontero, and V. Belle. Dynamics of the intrinsically disordered protein CP12 in its association with GAPDH in the green alga Chlamydomonas reinhardtii: a fuzzy complex, Mol Biosyst. 9 (2013) 2869-2876.

[73] J. P. Barnes, Z. Liang, H. S. McHaourab, J. H. Freed, and W. L. Hubbell. A multifrequency electron spin resonance study of T4 lysozyme dynamics, Biophysical Journal. 76 (1999) 3298-3306.

[74] L. Mainali, M. Raguz, and W. K. Subczynski. Phases and domains in sphingomyelin-cholesterol membranes: structure and properties using EPR spin-labeling methods, Eur Biophys J. 41 (2012) 147-159.

[75] L. Mainali, M. Raguz, and W. K. Subczynski. Phase-separation and domain-formation in cholesterol-sphingomyelin mixture: pulse-EPR oxygen probing, Biophys J. 101 (2011) 837-846. 